\title{
Piezo Electricity Transducers for Energy Harvesting
}

\author{
T Himavarsha', Yachauaram Yeshwant ${ }^{2}$, M Srinivas Rao ${ }^{3}$, Goli Sahithya ${ }^{4}$ \\ Dept of ECE, GITAM University, Hyderabad, India ${ }^{1,2,3,4}$
}

\begin{abstract}
As the end of the fossil fuels. Fossil fuel phase out is the proposed energy transition beyond fossil fuels through multiple means, including transport electrification, fossil fuel divestment, decommissioning of operating fossil fuel-fired power plants and prevention of construction of new fossil fuel fired power stations. Its purpose is to reduce air pollution, mining tragedies and greenhouse gas emissions which cause climate change. A move to many forms of renewable energy or nuclear energy is involved in shifting away from fossil fuels. We are considering piezoelectric crystals to harvest energy. Electricity produced is stored in a LA battery. The battery acts a source for the local (bulb \& fan in this case). The Arduino board is used to control the bulb \& fan with wireless Bluetooth connection with the help of Bluetooth module. In this paper wireless connection is used to efficiently use the energy produced by the piezoelectric effect and stored in the lead acid battery for further \&efficient use.
\end{abstract}

Keywords: Lead Acid (LA), Fossil fuel, Electricity, Energy Harvesting.

\section{INTRODUCTION}

For an alternate method to generate electricity there are number of methods by which electricity can be produced, out if such methods footstep energy generation can be an effective method to generate electricity.

Walking is the most common activity in human life. When a person walks, he loses energy to the road surface in the form of impact, vibration, sound etc., due to the transfer of his weight on to the road surface, through foot falls on the ground during every step. This energy can be tapped and converted in the usable form such as in electrical form. This device, if embedded in the footpath, can convert foot impact energy into electrical form.

Human-powered transport has been in existence since time immemorial in the form of walking, running and swimming. However modern technology has led to machines to enhance the use of human-power in more efficient manner. In this context, pedal power is an excellent source of energy and has been in use since the nineteenth century making use of the most powerful muscles in the body. Ninety-five percent of the exertion put into pedal power is converted into energy. Pedal power can be applied to a wide range of jobs and is a simple, cheap, and convenient source of energy. However, human kinetic energy can be useful in a number of ways but it can also be used to generate electricity based on different approaches and many organizations are already implementing human powered technologies to generate electricity to power small electronic appliances.

BASICPRINCIPLE: The downward movement of the plate results in rotation of the shaft of an electrical alternator fitted in the device, to produce electrical energy. The top plate reverts back to its original position due to negating springs provided in the device.

\section{INITIATIVE}

Working on the idea to harness human locomotion power, MIT (USA) architecture students James Graham and Thaddeus Jusczyk recently unveiled what they're calling the "Crowd Farm," a setup that would derive energy from pounding feet in crowded places. This technology is a proposal to harness human power as a source of sustainable energy. Population of India and mobility of its masses will turn into boon in generating electricity from its (population's) footsteps. Human locomotion in over crowded subway stations, railway stations, bus stands, airports, temples or rock concerts thus can be converted to electrical energy with the use of this promising technology.

The technology would turn the mechanical energy of people walking or jumping into a source of electricity. The students' test case, displayed at the Venice Biennale and in a train station in Torino, Italy, was a prototype stool that exploits the passive act of sitting to generate power. The weight of the body on the seat causes a flywheel to spin, which powers a dynamo that, in turn, lights four LEDs. In each case, there would be a sub-flooring system consisting of independent blocks. When people walk across this surface, the forces they impart will cause the blocks to slip slightly, and a dynamo would convert the energy in those movements into electric current. Students say that moving from this Proof-of-concept device to a large-scale Crowd Farm would be expensive, but it certainly sounds a great option. 


\section{PARTS}

SENSOR: A sensor is a device that measures a physical quantity and converts it into a signal which can be read by an observer or by an instrument. For example, mercury converts the measured temperature into expansion and contraction of a liquid which can be read on a calibrated glass tube. At thermocouple converts temperature to an output voltage which can be read by a voltmeter. For accuracy, most sensors are calibrated against known standards.

PIEZOELECTRIC SENSOR:A piezoelectric sensor is a device that uses the piezoelectric effect to measure pressure, acceleration, strain or force by converting them to an electrical signal.

Piezoelectric sensors have proven to be versatile tools for the measurement of various processes. They are used for quality assurance, process control and for research and development in many different industries it was only in the 1950s that the piezoelectric effect started to be used for industrial sensing applications. Since then, this measuring principle has been increasingly used and can be regarded as a mature technology with an outstanding inherent reliability. It has been successfully used in various applications, such as in medical, aerospace, nuclear instrumentation, and as a pressure sensor in the touch pads of mobile phones. In the automotive industry, piezoelectric elements are used to monitor combustion when developing internal combustion engines. The sensors are either directly mounted into additional holes into the cylinder head or the spark/glow plug is equipped with a built in miniature piezoelectric sensor.

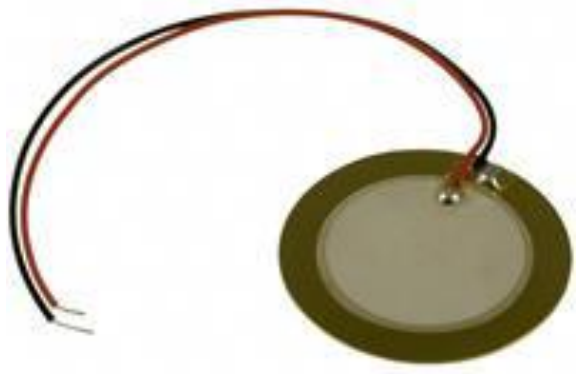

Fig 3.1 Piezoelectric crystal

The rise of piezoelectric technology is directly related to a set of inherent advantages. The high modulus of elasticity of many piezoelectric materials is comparable to that of many metals and goes up to $10 \mathrm{e} 6 \mathrm{~N} / \mathrm{m}^{2}$ [Even though piezoelectric sensors are electromechanical systems that react to compression, the sensing elements show almost zero deflection. This is the reason why piezoelectric sensors are so rugged, have an extremely high natural frequency and an excellent linearity over a wide amplitude range. Additionally, piezoelectric technology is insensitive to electromagnetic fields and radiation, enabling measurements under harsh conditions. Some materials used (especially gallium phosphate or tourmaline) have an extreme stability even at high temperature, enabling sensors to have a working range of up to $1000^{\circ} \mathrm{C}$. Tourmaline shows pyro electricity in addition to the piezoelectric effect; this is the ability to generate an electrical signal when the temperature of the crystal changes. This effect is also common to piezo ceramic materials. One disadvantage of piezoelectric sensors is that they cannot be used for truly static measurements. A static force will result in a fixed amount of charges on the piezoelectric material. While working with conventional readout electronics, imperfect insulating materials, and reduction in internal sensor resistance will result in a constant loss of electrons, and yield a decreasing signal.

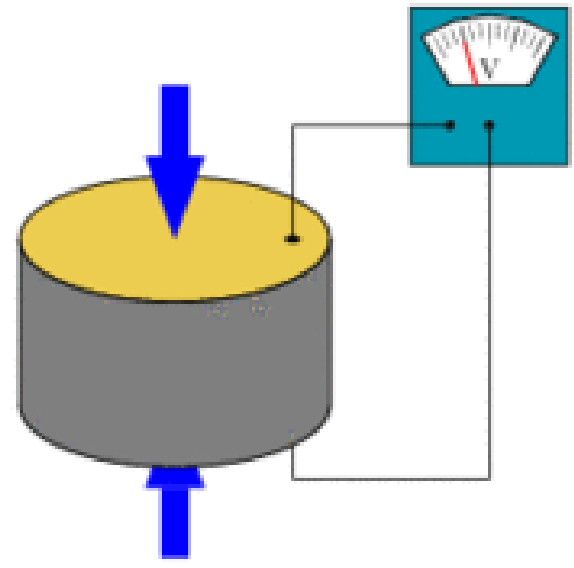

Fig 3.2 Piezoelectric sensor 


\section{International Journal of Innovative Research in Electrical, Electronics, Instrumentation and Control Engineering}

\section{ISO 3297:2007 Certified}

Vol. 5, Issue 6, June 2017

Elevated temperatures cause an additional drop in internal resistance and sensitivity. The main effect on the piezoelectric effect is that with increasing pressure loads and temperature, the sensitivity is reduced due to twinformation. While quartz sensors need to be cooled during measurements at temperatures above $300^{\circ} \mathrm{C}$, special types of crystals like GaPO4 gallium phosphate do not show any twin formation up to the melting point of the material.

BATTERY: Battery (electricity), an array of electrochemical cells for electricity storage, either individually linked or individually linked and housed in a single unit. An electrical battery is a combination of one or more electrochemical cells, used to convert stored chemical energy into electrical energy. Batteries may be used once and discarded, or recharged for years as in standby power applications. Miniature cells are used to power devices such as hearing aids and wristwatches; larger batteries provide standby power for telephone exchanges or computer data centers.

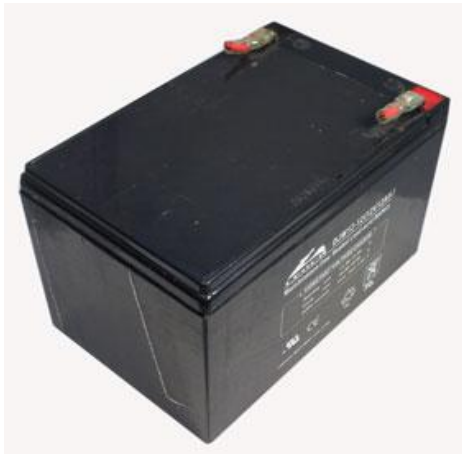

Fig: 3.3 Lead acid battery

Lead-acid batteries are the most common in PV systems because their initial cost is lower and because they are readily available nearly everywhere in the world. There are many different sizes and designs of lead-acid batteries, but the most important designation is that they are deep cycle batteries. Lead-acid batteries are available in both wet-cell (requires maintenance) and sealed no-maintenance versions. Lead acid batteries are reliable and cost effective with an exceptionally long life. The Lead acid batteries have high reliability because of their ability to withstand overcharge, over discharge vibration and shock. The use of special sealing techniques ensures that our batteries are leak proof and non- spoilable. The batteries have exceptional charge acceptance, large electrolyte volume and low self-discharge, which make them ideal as zero- maintenance batteries lead acid batteries are manufactured/ tested using CAD (Computer Aided Design). These batteries are used in Inverter \& UPS Systems and have the proven ability to perform under extreme conditions. The batteries have electrolyte volume, use PE Separators and are sealed in sturdy containers, which give them excellent protection against leakage and corrosion.

Features:

1) Manufactured/tested using CAD

2) Electrolyte volume

3) PE Separators

4) Protection against leakage

BATTERY CONNECTIONS: Lead-acid batteries are normally available in blocks of $2 \mathrm{~V}, 6 \mathrm{~V}$ or $12 \mathrm{~V}$. In most cases, to generate the necessary operating voltage and the capacity of the batteries for the Solar Inverter, many batteries have to be connected together in parallel and/or in series. Following three examples are shown:

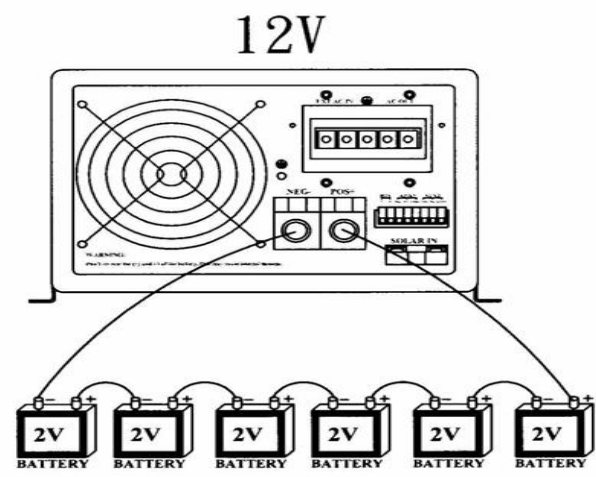

Fig: 3.4 Battery parallel connections 


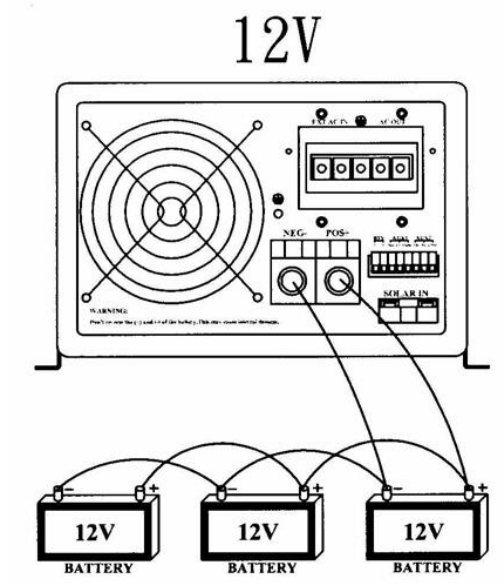

Fig: 3.5 Battery Series Connection

RECTIFIER: The output from the transformer is fed to the rectifier. It converts A.C. into pulsating D.C. The rectifier may be a half wave or a full wave rectifier. In this project, a bridge rectifier is used because of its merits like good stability and full wave rectification. The Bridge rectifier is a circuit, which converts an ac voltage to dc voltage using both half cycles of the input ac voltage. The Bridge rectifier circuit is shown in the figure. The circuit has four diodes connected to form a bridge. The ac input voltage is applied to the diagonally opposite ends of the bridge. The load resistance is connected between the other two ends of the bridge.

For the positive half cycle of the input ac voltage, diodes D1 and D3 conduct, whereas diodes D2 and D4 remain in the OFF state. The conducting diodes will be in series with the load resistance RL and hence the load current flows through RL.

For the negative half cycle of the input ac voltage, diodes D2 and D4 conduct whereas, D1 and D3 remain OFF. The conducting diodes D2 and D4 will be in series with the load resistance RL and hence the current flows through RL in the same direction as in the previous half cycle. Thus a bi-directional wave is converted into a unidirectional wave.

VOLTAGE REGULATOR: As the name itself implies, it regulates the input applied to it. A voltage regulator is an electrical regulator designed to automatically maintain a constant voltage level.

In this project, power supply of $5 \mathrm{~V}$ and $12 \mathrm{~V}$ are required. In order to obtain these voltage levels, 7805 and 7812 voltage regulators are to be used. The first number 78 represents positive supply and the numbers 05,12 represent the required output voltage levels. These regulators can provide local on-card regulation, eliminating the distribution problems associated with single point regulation. Each type employs internal current limiting, thermal shut-down and safe area protection, making it essentially indestructible.

If adequate heat sinking is provided, they can deliver over $1 \mathrm{~A}$ output current. Although designed primarily as fixed voltage regulators, these devices can be used with external components to obtain adjustable voltage and currents.

UNIDIRECTIONAL CURRENT CONTROLLER: As name indicates this circuit allows only one direction current flowing. There are following some devices allow on unidirectional current.

1) Diode

2) Thyristors

In this project we are going to use diode as Unidirectional Current control device. As we are already familiar with the most common function of a diode is to allow an electric current to pass in one direction (called the diode's forward direction). While blocking current in the opposite direction (the reverse direction). Thus, the diode can be thought of as an electronic version of a check valve. The diode used in this project is $\mathrm{D}=1 \mathrm{~N} 4007$.

MICROCONTROLLER AT89S52: In this project we are going to use the Microcontroller AT89S52. Features are At89s52:

1) ROM - 8 K bytes

2) RAM - 256 bytes

3) Timer - 3

4) I/O pins - 32

5) Serial port - 1

6) Interrupt sources - 8 
Here we are using AT89S52 to display the amount of battery get charged whenever we place our foot on piezoelectric transducer.

At89s52 Microcontroller Configuration:

1. Pin 1 to 8 (Port 1) is an 8-bit parallel port of a two-way (bidirectional) that can be used for different purposes (general purpose). Pin 9 is a pin reset, reset is active if a high ration.

2. P3.0 (10): RXD (serial port data receiver)

3. P3.1 (11): TXD (serial port data sender)

4. P3.2 (12): INT0 (external interrupt 0 input, active low)

5. P3.3 (13): INT1 (external an interrupt input, active low)

6. P3.4 (14): T0 (external input timer / counter 0)

7. P3.5 (15): T1 (external input timer / counter 1)

8. P3.6 (16): WR (Write, active low) control signal from port 0 write data to memory and input-output data externally.

9. P3.7 (17): RD (Read, active low) control signal of the reading of input-output data memory external to the port 0. XTAL pin 18 as the second, the output is connected to the crystal oscillator. XTAL pin 19 as the first, high input to the oscillator, connected to the crystal.

10. Pin 20 as Vss, is connected to 0 or ground on the circuit.

11. Pin 21 to 28 (Port 2) is 8 bits parallel ports in both directions. This port sends the address byte when accessing external memory is carried on.

12. Pin 29 as the PSEN (Program Store Enable) is the signal used for reading, move the program the external memory (ROM / EPROM) to microcontroller (active low).

13. Pin 30 as the ALE (Address Latch Enable) to hold down the address for accessing external memory.

14. Pin 31 as the EA (External Access) to select the memory to be used, the internal program memory $(\mathrm{EA}=\mathrm{Fcc})$ or external program memory $(\mathrm{EA}=\mathrm{Vss}$ ), Pin 32 to 39 (Port 0) is an 8-bit parallel port in both directions. Under which functions as a multiplexed address data to access an external program and data memory.

15. Pin 40 as Fcc, connected to $+5 \mathrm{~V}$ as a ration to the microcontroller. All single chips in the family division of MCS51 have the address space to programs and data.

ADC: An analog-to-digital converter (abbreviated ADC, A/D or A to D) is a device that converts a continuous quantity to a discrete time digital representation. An ADC may also provide an isolated measurement. The reverse operation is performed by a digital-to-analog converter (DAC).

Typically, an ADC is an electronic device that converts an input analog voltage or current to a digital number proportional to the magnitude of the voltage or current. However, some non-electronic or only partially electronic devices, such as rotary encoders, can also be considered ADCs

Features:

1. Easy interface to all microprocessors

2. Operates ratio metrically or with $5 \mathrm{VDC}$ or analog span

3. Adjusted voltage reference

4. No zero or full-scale adjust required

5. 8-channel multiplexer with address logic

6. $0 \mathrm{~V}$ to VCC input range

7. Outputs meet TTL voltage level specifications

Key Specifications:

1. Resolution 8 Bits

2. Total Unadjusted Error $\pm 1 / 2$ LSB and \pm 1 LSB

3. Single Supply 5 V DC

4. Low Power $15 \mathrm{~mW}$

5. Conversion Time $100 \mu \mathrm{s}$

INVERTER: An inverter is an electrical device that converts direct current (DC) to alternating current (AC); the converted $\mathrm{AC}$ can be at any required voltage and frequency with the use of appropriate transformers, switching, and control circuits.

Solid-state inverters have no moving parts and are used in a wide range of applications, from small switching power supplies in computers, to large electric utility high-voltage direct current applications that transport bulk power. Inverters are commonly used to supply AC power from DC sources such as solar panels or batteries.

There are two main types of inverter. The output of a modified sine wave inverter is similar to a square wave output except that the output goes to zero volts for a time before switching positive or negative. It is simple and low cost and is 
compatible with most electronic devices, except for sensitive or specialized equipment, for example certain laser printers.

A pure sine wave inverter produces a nearly perfect sine wave output $(<3 \%$ total harmonic distortion) that is essentially the same as utility-supplied grid power. Thus it is compatible with all AC electronic devices. This is the type used in grid-tie inverters. Its design is more complex, and costs 5 or 10 times more per unit power.

The electrical inverter is a high-power electronic oscillator. It is so named because early mechanical AC to DC converters was made to work in reverse, and thus were "inverted", to convert DC to AC. The inverter performs the opposite function of a rectifier.

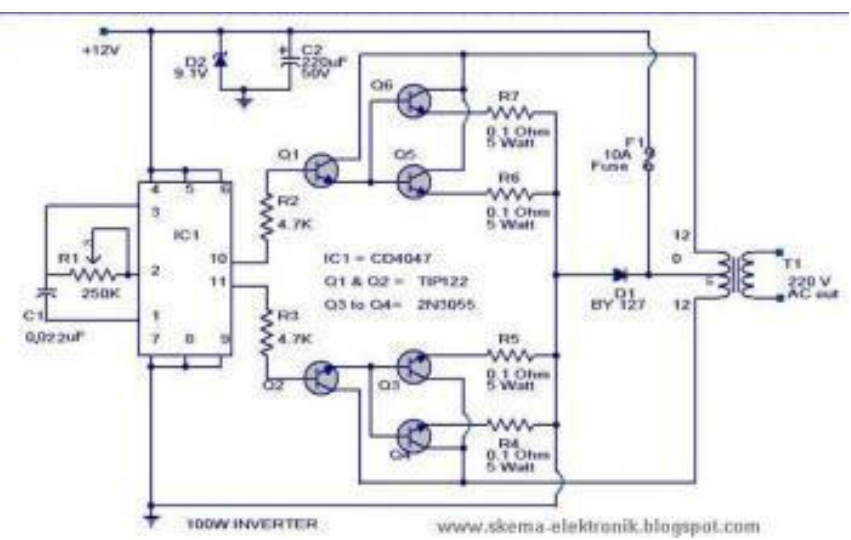

Fig: 3.6 100W invertor

The IC1 Cd4047 wired as an Astable multivibrator produces two 180 degree out of phase 1/50 Hz pulse trains. These pulse trains are preamplifiers by the two TIP122 transistors. The out puts of the TIP 122 transistors are amplified by four $2 \mathrm{~N} 3055$ transistors (two transistors for each half cycle) to drive the inverter transformer. The $220 \mathrm{~V}$ AC will be available at the secondary of the transformer.

Nothing complex just the elementary inverter principle and the circuit works great for small loads like a few bulbs or fans.

\section{VOLTAGE SAMPLER (SAMPLE \&HOLD CIRCUIT):}

Sample-and-hold $(\mathrm{S} / \mathrm{H})$ is an important analog building block with many applications, including analog-to-digital converters (ADCs) and switched-capacitor filters. The function of the $\mathrm{S} / \mathrm{H}$ circuit is to sample an analog input signal and hold this value over a certain length of time for subsequent processing.

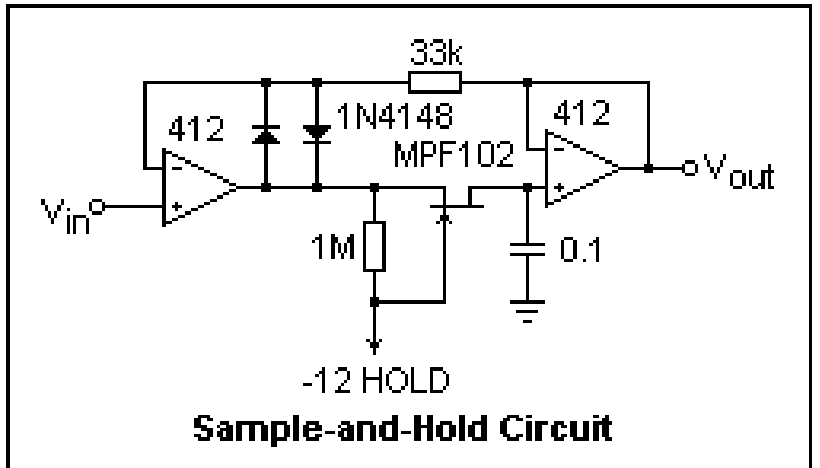

Fig 3.7 Sample and hold circuit

Taking advantages of the excellent properties of MOS capacitors and switches, traditional switched capacitor techniques can be used to realize different $\mathrm{S} / \mathrm{H}$ circuits [1]. The simplest $\mathrm{S} / \mathrm{H}$ circuit in MOS technology is shown in Figure 1, where Vin is the input signal, M1 is an MOS transistor operating as the sampling switch, Ch is the hold capacitor, ck is the clock signal, and Vout is the resulting sample-and-hold output signal.

In the simplest sense, a S/H circuit can be achieved using only one MOS transistor and one capacitor. The operation of this circuit is very straightforward. Whenever ck is high, the MOS switch is on, which in turn allows Vout to track Vin. On the other hand, when ck is low, the MOS switch is off. 
UGC Approved Journal

IJIREEICE

Vol. 5, Issue 6, June 2017

During this time, Ch will keep Vout equal to the value of Vin at the instance when ck goes low. CMOS Sample-andHold Circuits Page .Unfortunately, in reality, the performance of this $\mathrm{S} / \mathrm{H}$ circuit is not as ideal as described above. The next section of this paper explains two major types of errors, charge injection.

And clock feed through, that are associated with this $\mathrm{S} / \mathrm{H}$ implementation. The section after that presents three new $\mathrm{S} / \mathrm{H}$ techniques, all of which try to minimize the errors caused by charge injection and/or clock feed through.

As we know the pressure is directly proportional to amount of power generated

$\mathrm{P} \alpha \mathrm{Wt}$

Here we take the constant of proportionality as $K$, then the equation becomes

$\mathrm{P}=\mathrm{K} \mathrm{Wt}$

Where,

K- Constant of proportionality

Wt-weight

P-power

We know that for wt $=50 \mathrm{~kg}$, we get the value of voltage $\mathrm{V}=4 \mathrm{v}$ and $\mathrm{I}=0.015 \mathrm{~A}$

Then $\mathrm{P}=\mathrm{V}^{*} \mathrm{I}=4 * 0.015=0.06 \mathrm{w}$, means we can say that for $50 \mathrm{~kg}$ we get power

$(\mathrm{P})=0.06 \mathrm{w}$

From this we can find the value of $\mathrm{K}$

$\mathrm{K}=\mathrm{P} / \mathrm{wt}=0.06 / 50=0.0012$

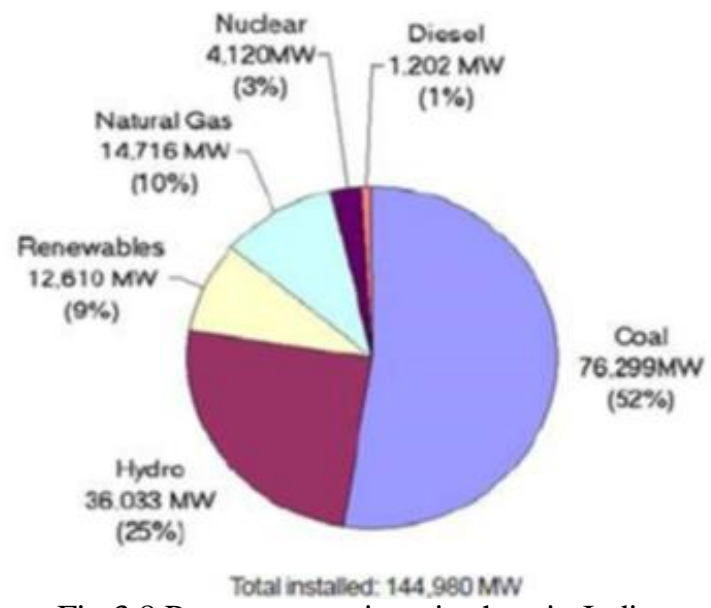

Fig 3.8 Power generation pie chart in India

BLOCK DIAGRAM: These is the basic block diagram for the energy harvesting. In these paper, we tried to improve the circuit by including the battery for proper storage and using of the harvested energy further and Arduino board with the bluetooth module for wireless connection and efficient usage of the harvested energy by the piezo electric crystal.

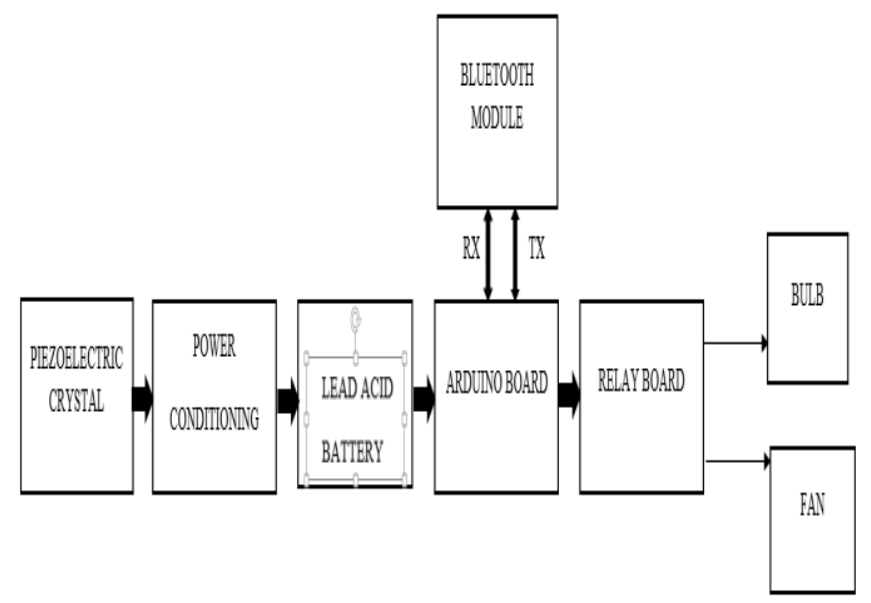

Fig 3.9 Block diagram 
UGC Approved Journal

IJIREEICE

\section{International Journal of Innovative Research in} Electrical, Electronics, Instrumentation and Control Engineering

ISO 3297:2007 Certified

Vol. 5, Issue 6, June 2017

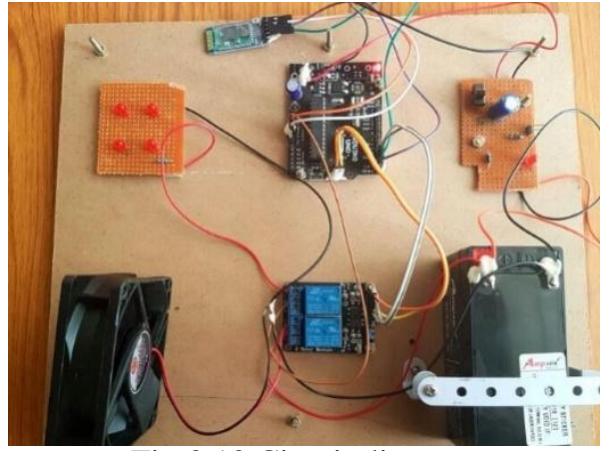

Fig 3.10 Circuit diagram

\section{CONCLUSION}

1. The project "POWER GENERATION USING FOOT STEP" is successfully tested and implemented which is the best economical, affordable energy solution to common people.

2. This can be used for many applications in rural areas where power availability is less or totally absence As India is a developing country where energy management is a big challenge for huge population. By using this project we can drive both A.C. as well as D.C loads according to the force we applied on the piezo electric sensor.

\section{REFERENCES}

[1] Energy Harvesting using Piezoelectricity Renewable and sustainable energy conversion using piezoelectric transducers 2015 International Conference on Energy Systems and Applications (ICESA 2015)Dr. D. Y. Patil Institute Engineering and Technology, Pune, India 30 Oct - 01 Nov, 2015

[2] Energy scavenging with shoe-mounted piezoelectrics -N.S.shenek; J.A.Paradiso

[3] A review of three-pase improved Ac-Dc converts - B.Singh; B.N Singh,A.Chandra

[4] Simulation of piezos by two-three dimension finite elements- R.Lech

[5] z-bus allocation - A.J.Conejo; F.D.Galina 